Rev. Int. Contam. Ambie. 32 (4) 445-453, 2016

DOI: 10.20937/RICA.2016.32.04.08

\title{
CRECIMIENTO, NUTRICIÓN Y RENDIMIENTO DE CALABACITA CON FERTILIZACIÓN BIOLÓGICA Y MINERAL
}

\author{
Arturo DÍAZ FRANCO, Manuel ALVARADO CARRILLO \\ Florencia ALEJANDRO ALLENDE y Flor Elena ORTIZ CHÁIREZ*
}

Instituto Nacional de Investigaciones Forestales Agrícolas y Pecuarias. Carretera Matamoros Reynosa, kilómetro 61, Tamaulipas, México, C.P. 88900

*Autor para correspondencia: ortiz.flor@inifap.gob.mx

(Recibido febrero 2015; aceptado enero 2016)

Palabras clave: productividad, gallinaza, micorriza arbuscular, Cucurbita pepo

\section{RESUMEN}

En la agricultura moderna el paradigma emergente es reducir o eliminar el uso de agroquímicos sintéticos que impactan de manera adversa al ambiente. Por ello, ahora se privilegia la práctica de fertilización biológica con productos naturales. El propósito del estudio fue conocer el efecto de la gallinaza y del hongo micorrízico arbuscular (HMA) Rhizophagus intraradices, comparado con la fertilización inorgánica 120-9000, en las características de la planta de calabacita (Cucurbita pepo) y su productividad, tanto en invernadero como en campo. En invernadero, la gallinaza y el HMA se aplicaron a razón de 3 y $5 \mathrm{~g} /$ maceta, en campo fue de $0.5 \mathrm{~kg} / 3.3 \mathrm{~kg}$ de semilla y $3 \mathrm{t} / \mathrm{ha}$, respectivamente. En 2012 se midió el índice de clorofila (SPAD), la altura de la planta, las biomasas foliar y radical y la colonización micorrízica. Durante 2012 y 2013, se evaluó el índice de clorofila, la altura de la planta, el diámetro y el peso del fruto y el rendimiento. Además, en 2013 se determinó el contenido foliar de $\mathrm{P}, \mathrm{Cu}, \mathrm{Zn}, \mathrm{Mn}$, $\mathrm{Fe}, \mathrm{Ca}$ y Mg. En invernadero, en general la fertilización inorgánica, la gallinaza y el HMA, superaron al testigo absoluto en todas las variables evaluadas. Los tratamientos con el HMA tuvieron en promedio 59 \% de colonización micorrízica. En campo, los valores mayores de clorofila (37.8 SPAD), de la altura de la planta $(68.3 \mathrm{~cm})$, del peso del fruto $(220 \mathrm{~g})$ y del rendimiento $(31.3 \mathrm{t} / \mathrm{ha})$, se registraron en $2012 \mathrm{y}$ estuvieron asociados a una mayor precipitación. Los resultados de 2012 y 2013 indicaron que la mayor altura de la planta se registró con la fertilización inorgánica, aunque no hubo diferencias entre gallinaza, HMA y fertilización inorgánica para clorofila, diámetro y peso del fruto, así como rendimiento total. Además, el contenido foliar de $\mathrm{P}, \mathrm{Cu}, \mathrm{Zn}$, $\mathrm{Mn}, \mathrm{Fe}, \mathrm{Ca}$ y $\mathrm{Mg}$, fue semejante en los mismos tratamientos. Los resultados parecen indicar que la gallinaza y la inoculación del HMA registraron características de planta, fruto y rendimiento en calabacita, equiparables con la fertilización mineral.

Key words: productivity, chicken manure, arbuscular mycorrhiza, Cucurbita pepo

\begin{abstract}
In modern agriculture the emerging paradigm is to reduce or eliminate the use of synthetic agrochemicals that adversely impact the environment. Therefore, the use
\end{abstract}


of natural products such as biological fertilization is now privileged. The purpose of this study was to determine the effect of chicken manure and inoculation with arbuscular mycorrhizal fungus (AMF) Rhizophagus intraradices, compared with inorganic fertilizer 120-90-00, on characteristics of squash plant (Cucurbita pepo) and its productivity, under greenhouse and field conditions. In greenhouse, the chicken manure and the AMF were applied at the rate of 3 and $5 \mathrm{~g} /$ pot, in field they were $0.5 \mathrm{~kg} / 3.3 \mathrm{~kg}$ of seed and $3 \mathrm{t} / \mathrm{ha}$, respectively. Chlorophyll index (SPAD), plant height, foliar and radical biomass, and mycorrhizal colonization were measured in the greenhouse. In field, 2012 and 2013, the variables evaluated were chlorophyll, plant height, diameter and weight of fruit, as well as yields. In addition, in 2013 foliar contents of $\mathrm{P}, \mathrm{Cu}, \mathrm{Zn}$, $\mathrm{Mn}, \mathrm{Fe}, \mathrm{Ca}$ and $\mathrm{Mg}$ were determined. In greenhouse, in general, inorganic fertilizer, chicken manure and AMF, exceeded the absolute control group in all of variables evaluated. Treatments with AMF had an average of $59 \%$ of mycorrhizal colonization. In field, the greatest chlorophyll values (37.8 SPAD), plant height $(68.3 \mathrm{~cm})$, weight of fruit (220 g) and yield (31.3 t/ha), were recorded in 2012, and they were associated with greater rainfall. Results of 2012 and 2013 indicated that larger plant height was recorded with inorganic fertilizer, although there were no significant differences between chicken manure, AMF and inorganic fertilizer for chlorophyll, diameter and weight of fruit, and total yield. Also, foliar contents of $\mathrm{P}, \mathrm{Cu}, \mathrm{Zn}, \mathrm{Mn}, \mathrm{Fe}, \mathrm{Ca}$ and $\mathrm{Mg}$, were similar in the same treatments. The results seem to indicate that chicken manure and AMF inoculation recorded in squash plant characteristics, fruit and yields, are comparable with that of mineral fertilization.

\section{INTRODUCCIÓN}

En la actualidad, la investigación biotecnológica está mostrando especial interés en la producción hortícola con enfoque sostenible debido a que el uso excesivo de fertilizantes sintéticos ha tenido como resultado la contaminación del ambiente, el decremento de la biodiversidad, la degradación de los suelos y el incremento de los costos de producción. Adesemoye y Kloepper (2009) señalaron que se ha percibido la necesidad de implementar métodos que permitan, entre otras cosas, mejorar la eficiencia de los cultivos, disminuir la tasa de uso de fertilizantes sintéticos, mitigar los efectos adversos sobre el ambiente y aumentar las ganancias por área cultivada.

La fertilización biológica se basa en la utilización de insumos naturales dentro de los que se encuentran los abonos orgánicos, compostas, biosólidos y microorganismos como hongos y bacterias, para mejorar la absorción de nutrientes en la rizósfera, producir estimulantes de crecimiento para las plantas, mejorar la estabilidad del suelo, biodegradar sustancias, reciclar nutrientes y favorecer sinergias microbianas, entre otros. Adicionalmente, su uso permite mejorar la productividad por área cultivada en corto tiempo, consumir menores cantidades de energía, mitigar la contaminación del suelo y del agua, incrementar la fertilidad del suelo y favorecer el control biológico de fitopatógenos (Carbajal y Mera 2010). En particular, los hongos micorrízicos arbusculares (HMA) durante la simbiosis aportan beneficios como la promoción del crecimiento y mayor nutrición mineral de la planta cultivada (Carpio et al. 2005, Díaz et al. 2013), tolerancia a patógenos del suelo (Graham 2001, Tahat et al. 2010) y a condiciones abióticas adversas (Davies et al. 2002, Rabie 2005). Otra opción viable es utilizar abonos orgánicos ya que tienen el potencial de ser una fuente de nutrientes económica y de gran eficacia en la nutrición de los cultivos. La descomposición o mineralización de cualquier abono orgánico una vez incorporado al suelo es afectado por diversos factores como la humedad, la temperatura, la aireación, el tipo de material orgánico, la cantidad aplicada y el tipo de suelo, entre otros (Eghball et al. 2002, Flores et al. 2007). Dentro de los abonos orgánicos, la gallinaza y el estiércol de cerdo son los más ricos desde el aspecto nutrimental y de mayor liberación de nutrimentos en el primer año (Hue y Silva 2000).

Se ha indicado que la práctica de fertilización biológica podría sustituir o disminuir a la fertilización sintética y traer consigo efectos benéficos desde las perspectivas económica, social y ecológica (Adesemoye y Kloepper 2009, Carbajal y Mera 2010). Se han realizado diversos estudios para conocer los efectos de los abonos orgánicos (Desgan y Bozkoylu 2007, Rodríguez et al. 2009, Bouajila et al. 2011, Velázquez et al. 2014) y de bioinoculantes (Davies et al. 2002, Carpio et al. 2005, Terry et al. 2005, Díaz et al. 2013) en los cultivos, no obstante, 
es limitada la información relacionada con la efectividad independiente o asociada entre los abonos orgánicos y la inoculación con hongos formadores de micorrizas. Por lo anterior, el propósito del presente estudio fue conocer el efecto de la gallinaza y del HMA Rhizophagus intraradices, en combinación e independientes, sobre las características de la planta de calabacita (Cucurbita pepo) y su productividad.

\section{MATERIALES Y MÉTODOS}

El estudio se desarrolló durante 2012 y 2013 en condiciones de invernadero y campo en el Campo Experimental Río Bravo (CERIB), Instituto Nacional de Investigaciones Forestales, Agrícolas y Pecuarias (INIFAP), Río Bravo, Tamaulipas, México, ubicado a $25^{\circ} 57^{\prime} \mathrm{LN}, 98^{\circ} 01^{\prime} \mathrm{LO}, 25 \mathrm{msnm}$, con clima semicálido y suelos vertisoles. Las características particulares de los suelos en presiembra manejados en el estudio están descritas en el cuadro I. En todos los casos se utilizó el híbrido de calabacita tipo zuchini cultivar Spineless Perfection.

Experimento en invernadero. El primer experimento se estableció en un invernadero de baja tecnología del CERIB. El suelo utilizado se mezcló con $20 \%$ (v/v) de termolita, se depositó y cubrió con lona de plástico y se esterilizó con bromuro de metilo. Después de cinco días se llenaron bolsas de plástico negro con volumen de $7 \mathrm{~kg}$ de capacidad. Los tratamientos evaluados fueron: 1) gallinaza procesada y deshidratada Meyfer ${ }^{\circledR}$ con las siguientes características: $4.64 \%$ nitrógeno $(\mathrm{N})$ total, $5.42 \% \mathrm{P}, 3.13 \%$ de potasio $(\mathrm{K})$, $338 \mathrm{mg} / \mathrm{kg}$ de cobre $(\mathrm{Cu}), 6588 \mathrm{mg} / \mathrm{kg}$ de hierro $(\mathrm{Fe})$, $799 \mathrm{mg} / \mathrm{kg}$ de manganeso (Mn), $568 \mathrm{mg} / \mathrm{kg}$ de zinc (Zn), 42.1 \% de materia orgánica (M.O.), a razón de $5 \mathrm{~g} /$ maceta, 2) HMA $R$. intraradices (Micorriza INIFAP ${ }^{\circledR}$ con $\geq 60$ esporas $/ g$ ) inoculando al suelo con $3 \mathrm{~g} /$ maceta, 3) combinación de gallinaza Meyfer ${ }^{\circledR}$ y HMA $R$. intraradices, mismas dosis, 4) testigo fertilizado químicamente con 120-90-00 (Masabni
2012) y 5) testigo absoluto. El 9 de enero de 2012 se sembraron tres semillas por maceta y sólo se dejó una plántula. Cada tratamiento se estableció con 12 repeticiones, la unidad experimental estuvo constituida por una maceta, en un diseño con distribución completamente al azar. Se aplicaron riegos regulares y el cultivo se mantuvo hasta el estado de floración. En ese estado fenológico se midió in situ el índice de clorofila con lecturas en las hojas superiores de las plantas mediante determinaciones con un equipo Minolta SPAD $502^{\circledR}$, así como el diámetro del tallo, la altura de la planta y la biomasa foliar y radical seca, estas últimas a través del secado en estufa a $60{ }^{\circ} \mathrm{C}$ durante tres días. Antes del secado en estufa, secciones de $5 \mathrm{~g}$ de raíces frescas de cinco plantas se lavaron para eliminar el suelo. En éstas se determinó la colonización micorrízica en laboratorio, mediante la técnica de clareo con $10 \% \mathrm{KOH}$ y de tinción de azul tripano al 0.03 \% (Phillips y Hayman 1970), el porcentaje de estructuras fúngicas fue cuantificado con el método de Giovannetti y Mosse (1980). La información se sometió a análisis de varianza y la separación de medias fue mediante la prueba de Tukey $(\mathrm{p}<0.05)$.

Experimentos en campo. Las siembras se establecieron el 2 y 3 de marzo de 2012 y 2013, respectivamente, a una profundidad de aproximadamente $3 \mathrm{~cm}$ y densidad de $2-3$ plantas $/ \mathrm{m}$. Los tratamientos consistieron en: 1) la adición de gallinaza Meyfer ${ }^{\mathbb{B}}$ a razón de 3 t/ha (Ramírez 2012), incorporada en el fondo del surco antes del riego de presiembra, 2) el HMA $R$. intraradices (Micorriza INIFAP ${ }^{\circledR}$ ), inoculado en la semilla a dosis de $0.5 \mathrm{~kg} / \mathrm{ha}$ por cada $3.3 \mathrm{~kg}$ de semilla/ha, 3) combinación de gallinaza y HMA, mismas dosis y 4) testigo fertilizado con 120-90-00 a base de urea y fosfato monoamónico. La mitad de la urea y todo el fosfato se aplicaron al lado del surco en el momento de la siembra y el resto de la urea en floración. La cantidad de elementos mayores aportados con la adición de gallinaza fue de 119,56 y $80 \mathrm{~kg} / \mathrm{ha}$ de N, P y K, respectivamente (Hue y Silva 2000). Cada tratamiento se estableció en parcelas de cuatro

CUADRO I. PROPIEDADES QUÍMICAS Y FÍSICAS DE LOS SUELOS UTILIZADOS EN LOS ESTUDIOS

\begin{tabular}{|c|c|c|c|c|c|c|c|c|}
\hline \multicolumn{2}{|l|}{ Estudio } & \multirow{2}{*}{$\begin{array}{c}\text { M.O. } \\
(\%)\end{array}$} & \multirow{2}{*}{$\begin{array}{l}\mathrm{pH} \\
8.0\end{array}$} & \multirow{2}{*}{$\begin{array}{c}\begin{array}{c}\text { C.E. } \\
(\mathrm{dS} / \mathrm{m})\end{array} \\
1.26\end{array}$} & \multirow{2}{*}{$\begin{array}{c}\begin{array}{c}\mathrm{N} \\
(\mathrm{mg} / \mathrm{kg})\end{array} \\
18.1\end{array}$} & \multirow{2}{*}{$\begin{array}{c}\begin{array}{c}\mathrm{P} \\
(\mathrm{mg} / \mathrm{kg})\end{array} \\
17.3\end{array}$} & \multirow{2}{*}{$\begin{array}{c}\begin{array}{c}\mathrm{K} \\
(\mathrm{mg} / \mathrm{kg})\end{array} \\
1022\end{array}$} & \multirow{2}{*}{$\begin{array}{c}\text { Textura } \\
\text { Franco limoso }\end{array}$} \\
\hline Invernadero & 2012 & & & & & & & \\
\hline Campo & 2012 & 1.24 & 8.0 & 1.28 & 18.6 & 11.8 & 988 & Franco limoso \\
\hline & 2013 & 2.20 & 8.3 & 2.7 & 22.3 & 21.5 & 1673 & Arcilloso \\
\hline
\end{tabular}

M.O., materia orgánica, C.E., conductividad eléctrica 
surcos con $0.81 \mathrm{~m}$ de separación y $5 \mathrm{~m}$ de largo, las cuales se repitieron cuatro veces, en un diseño de bloques al azar. Se dispuso de un riego de presiembra y cuatro adicionales cada 15-20 días. Otras prácticas agronómicas como riegos, escardas y fitosanitarias se realizaron según las recomendaciones de Masabni (2010). De la estación meteorológica del CERIB se tomaron datos de temperatura y precipitación durante el ciclo biológico del cultivo en ambos años.

En 2013 se hicieron análisis foliares durante el estado de floración para determinar los contenidos de $\mathrm{P}, \mathrm{Cu}, \mathrm{Zn}, \mathrm{Mn}, \mathrm{Fe}, \mathrm{Ca}$ y Mg. El P se determinó mediante digestión ácida y el método molibdovanadato, mientras que los elementos menores, $\mathrm{Ca}$ y $\mathrm{Mg}$ a través de los métodos de digestión ácida y de absorción atómica (Plenecassangne et al. 1999).

En ambos años y después del segundo corte o cosecha, de cada parcela se midió el índice de clorofila (SPAD) tomado en 15 lecturas en las hojas superiores y la altura de cinco plantas. El rendimiento total del fruto se obtuvo de nueve cortes. Además, se determinó el diámetro y el peso del fruto. Para ello de cada corte se tomaron de forma aleatoria 10 frutos por parcela. Los datos de las variables evaluadas se sometieron a un análisis de varianza anual y combinado (2012-2013) y las diferencias entre medias fueron comparadas con la prueba de Tukey $(\mathrm{p} \leq 0.05)$.

\section{RESULTADOS Y DISCUSIÓN}

Experimento en invernadero. Los resultados indicaron que todos los tratamientos superaron al testigo absoluto en las características evaluadas en las plantas. No obstante, hubo variaciones en las respuestas. Los mayores valores del índice de clorofila se encontraron con el tratamiento que llevó fertilizante inorgánico. La mayor altura de la planta se registró con la combinación de gallinaza más HMA, aunque en el peso de biomasa foliar destacaron los tratamientos de fertilización inorgánica y la mezcla de gallinaza más HMA. En el diámetro de tallo y la biomasa radical, todos los tratamientos superaron de manera semejante al testigo absoluto. Los mayores porcentajes de colonización micorrízica fueron con el HMA y la combinación de gallinaza más HMA (Cuadro II). Los efectos sugieren que el HMA, la gallinaza y la combinación de ambos, fueron capaces de inducir un mayor desarrollo y crecimiento de las plantas de calabacita y en general compitieron con el tratamiento de fertilización química. En pepino desarrollado en agricultura protegida (Vásquez et al. 2014) los valores de área foliar, índice de clorofila y biomasa foliar fueron similares entre la fertilización sintética al $100 \%$ (200-200-100), comparada con la mitad del fertilizante más la inoculación de biofertilizante (complejo de bacterias promotoras de crecimiento y Glomus intraradices). Los datos apuntan a que la combinación gallinaza más HMA manifestó un efecto sinérgico para altura de la planta y la biomasa foliar, con esta última variable compitió con la fertilización inorgánica, en suelo estéril de invernadero.

Experimentos en campo. Los resultados obtenidos en 2012 indicaron que las variables evaluadas no mostraron diferencias significativas entre los tratamientos para los valores del índice de clorofila, diámetro de fruto, peso de fruto y rendimiento, los cuales promediaron 36.8 unidades SPAD, $47.4 \mathrm{~mm}$, $225 \mathrm{~g} \mathrm{y} 31.3 \mathrm{t} / \mathrm{ha}$ respectivamente. Por el contrario, la altura de planta fue afectada por los tratamientos, la mayor altura fue registrada con la fertilización inorgánica (76 cm; Cuadro III). En 2013, no se observaron diferencias significativas entre tratamientos en cada una de las variables, cuyos promedios fueron para clorofila 34.9 unidades SPAD, $56.4 \mathrm{~cm}$ en altura de planta, $46.7 \mathrm{~mm}$ de diámetro de fruto, $168 \mathrm{~g}$ de peso de fruto y $28.1 \mathrm{t} / \mathrm{ha}$ de rendimiento (Cuadro IV).

CUADRO II. INFLUENCIA DE LA GALLINAZA Y LA MICORRIZA ARBUSCULAR EN LAS CARACTERÍSTICAS DE LA PLANTA DE CALABACITA EN INVERNADERO

\begin{tabular}{lcccccc}
\hline Tratamiento & $\begin{array}{c}\text { Clorofila } \\
(\text { SPAD })\end{array}$ & $\begin{array}{c}\text { Diámetro de tallo } \\
(\mathrm{mm})\end{array}$ & $\begin{array}{c}\text { Altura de planta } \\
(\mathrm{cm})\end{array}$ & $\begin{array}{c}\text { Biomasa foliar } \\
(\mathrm{g})\end{array}$ & $\begin{array}{c}\text { Biomasa radical Colonización por HMA } \\
(\%)\end{array}$ \\
\hline Gallinaza $(\mathrm{G})$ & $28.0 \mathrm{~b}^{*}$ & $12.5 \mathrm{a}$ & $34.5 \mathrm{~b}$ & $71 \mathrm{~b}$ & $13.5 \mathrm{a}$ & $18.2 \mathrm{~b}$ \\
HMA & $29.9 \mathrm{~b}$ & $11.9 \mathrm{a}$ & $33.8 \mathrm{~b}$ & $67 \mathrm{~b}$ & $14.8 \mathrm{a}$ & $53.8 \mathrm{a}$ \\
$\mathrm{G}+$ HMA & $28.4 \mathrm{~b}$ & $12.7 \mathrm{a}$ & $39.7 \mathrm{a}$ & $85 \mathrm{a}$ & $13.8 \mathrm{a}$ & $64.2 \mathrm{a}$ \\
$120-90-00$ & $33.5 \mathrm{a}$ & $12.1 \mathrm{a}$ & $34.5 \mathrm{~b}$ & $92 \mathrm{a}$ & $13.0 \mathrm{a}$ & $0.0 \mathrm{c}$ \\
Testigo & $22.2 \mathrm{c}$ & $9.1 \mathrm{~b}$ & $29.0 \mathrm{c}$ & $41 \mathrm{c}$ & $5.6 \mathrm{~b}$ & $0.0 \mathrm{c}$ \\
Significancia F & 0.001 & 0.001 & 0.001 & 0.003 & 0.01 & 0.01 \\
\hline
\end{tabular}

*Valores unidos con la misma letra en la misma columna no difieren (Tukey, $\mathrm{p} \leq 0.05$ )

$\mathrm{SPAD}=$ Valor del índice de clorofila con medidor Minolta SPAD 502, G = Gallinaza, HMA = Hongos micorrízicos arbusculares 
El análisis combinado de ambos años indicó que no hubo interacción entre años y tratamientos, lo que significa que los factores son independientes. La respuesta de la calabacita fue diferente entre los dos años del estudio. Los valores de índice de clorofila (SPAD), la altura de la planta, el peso del fruto y el rendimiento total fueron superiores en las condiciones de 2012 (Cuadro V). Estos resultados obedecen a diferentes factores que pueden influir en el crecimiento y desarrollo de las plantas. Probablemente favorecieron variables como la precipitación de $175 \mathrm{~mm}$ registrada al inicio del periodo reproductivo (Fig. 1), las temperaturas ligeramente más cálidas durante 2012 (Fig. 2), además de las diferentes características de los suelos (Cuadro I), que pudieron promover mayor potencial de crecimiento y producción en el cultivo.

Los tratamientos no mostraron influencia en el índice de clorofila, ni en el diámetro, peso del fruto o rendimiento, solamente afectaron la altura de la

CUADRO III. CARACTERÍSTICAS DE PLANTA, FRUTO Y RENDIMIENTO DE CALABACITA MEDIANTE GALLINAZA, MICORRIZA ARBUSCULAR Y FERTILIZACIÓN INORGÁNICA EN 2012

\begin{tabular}{|c|c|c|c|c|c|}
\hline \multirow{2}{*}{ Tratamiento } & \multirow{2}{*}{$\begin{array}{l}\text { Clorofila } \\
\text { (SPAD) }\end{array}$} & \multirow{2}{*}{$\begin{array}{c}\text { Altura de } \\
\text { planta }(\mathrm{cm})\end{array}$} & \multicolumn{2}{|l|}{ Fruto } & \multirow{2}{*}{$\begin{array}{c}\text { Rendimiento } \\
\text { total }(\mathrm{t} / \mathrm{ha})\end{array}$} \\
\hline & & & Diámetro (mm) & Peso (g) & \\
\hline Gallinaza (3 t/ha) & 37.2 & $65.9 \mathrm{ab}$ & 46.7 & 228 & 32.9 \\
\hline HMA (0.5 kg/ha) & 36.6 & $68.9 \mathrm{ab}$ & 48.8 & 223 & 31.2 \\
\hline $\mathrm{G}+\mathrm{HMA}$ & 36.2 & $62.6 \mathrm{~b}$ & 47.3 & 222 & 30.3 \\
\hline $120-90-00$ & 37.4 & $76.0 \mathrm{a}$ & 46.9 & 227 & 31.1 \\
\hline Significancia F & 0.075 & 0.025 & 0.224 & 0.982 & 0.698 \\
\hline
\end{tabular}

${ }^{1}$ Valor del índice de clorofila con medidor Minolta SPAD 502

SPAD $=$ Valor del índice de clorofila con medidor Minolta SPAD 502, G = Gallinaza, HMA $=$ Hongos micorrízicos arbusculares

CUADRO IV. CARACTERÍSTICAS DE PLANTA, FRUTO Y RENDIMIENTO DE CALABACITA MEDIANTE GALLINAZA, MICORRIZA ARBUSCULAR Y FERTILIZACIÓN INORGÁNICA EN 2013

\begin{tabular}{|c|c|c|c|c|c|}
\hline \multirow[t]{2}{*}{ Tratamiento } & \multirow{2}{*}{$\begin{array}{l}\text { Clorofila } \\
\text { (SPAD) }\end{array}$} & \multirow{2}{*}{$\begin{array}{c}\text { Altura de } \\
\text { planta }(\mathrm{cm})\end{array}$} & \multicolumn{2}{|c|}{ Fruto } & \multirow{2}{*}{$\begin{array}{c}\text { Rendimiento } \\
\text { total }(\mathrm{t} / \mathrm{ha})\end{array}$} \\
\hline & & & Diámetro (mm) & Peso (g) & \\
\hline Gallinaza (3 t/ha) & 34.8 & 59.0 & 47.1 & 168 & 26.5 \\
\hline HMA (0.5 kg/ha) & 35.1 & 54.8 & 47.0 & 176 & 28.6 \\
\hline $\mathrm{G}+\mathrm{HMA}$ & 34.7 & 56.0 & 46.6 & 164 & 26.8 \\
\hline $120-90-00$ & 35.0 & 55.8 & 46.2 & 164 & 30.6 \\
\hline Significancia F & 0.231 & 0.403 & 0.91 & 0.825 & 0.555 \\
\hline
\end{tabular}

${ }^{1}$ Valor del índice de clorofila con medidor Minolta SPAD 502

$\mathrm{SPAD}=$ Valor del índice de clorofila con medidor Minolta SPAD 502, G $=$ Gallinaza, HMA = Hongos micorrízicos arbusculares

CUADRO V. PROMEDIO DE LAS CARACTERÍSTICAS DE PLANTA, FRUTO Y RENDIMIENTO DE FRUTO DE CALABACITA, CON FERILIZACIÓN INORGÁNICA Y BIOLÓGICA EN 2012 Y 2013

\begin{tabular}{lccccc}
\hline Año & Clorofila & Altura de & \multicolumn{2}{c}{ Fruto } & \multirow{2}{*}{$\begin{array}{c}\text { Rendimiento } \\
\text { total }(\mathrm{t} / \mathrm{ha})\end{array}$} \\
\cline { 5 - 6 } & $\left(\mathrm{SPAD}^{1}\right)$ & planta $(\mathrm{cm})$ & Diámetro $(\mathrm{mm})$ & Peso $(\mathrm{g})$ & \\
\hline 2012 & 36.8 & 68.3 & 47.4 & 224 & 31.3 \\
2013 & 34.9 & 56.4 & 46.7 & 168 & 28.1 \\
Significancia F & 0.030 & 0.001 & 0.24 & 0.01 & 0.021 \\
\hline
\end{tabular}

$\mathrm{SPAD}=$ Valor del índice de clorofila con medidor Minolta SPAD 502 


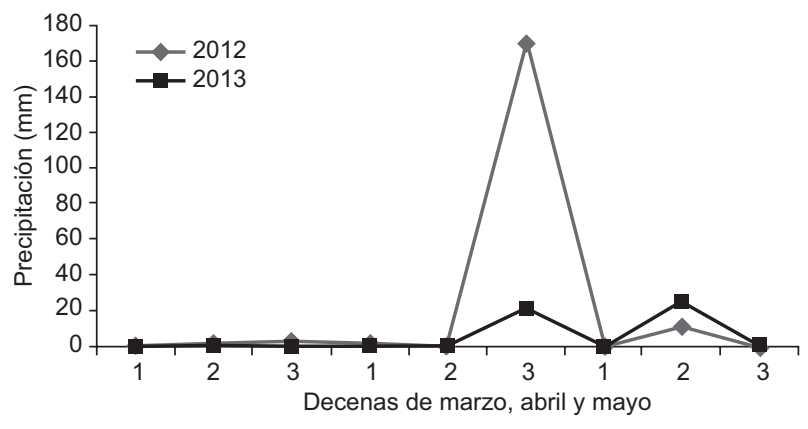

Fig. 1. Precipitación ( $\mathrm{mm}$ ) decenal registrada de marzo a mayo 2012 y 2013 en el Campo Experimental Río Bravo, Río Bravo, Tamaulipas, México

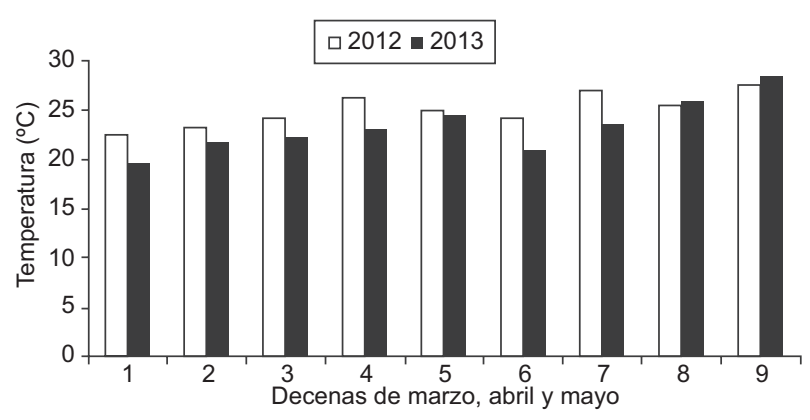

Fig. 2. Temperatura media $\left({ }^{\circ} \mathrm{C}\right)$ decenal registrada de marzo a mayo 2012 y 2013 en el Campo Experimental Río Bravo, Río Bravo, Tamaulipas, México

planta $(\mathrm{p}=0.040)$. La mayor altura de la planta se registró con la fertilización inorgánica, aunque estadísticamente semejante con gallinaza e inoculación micorrízica (Cuadro VI). En cambio, la mayor altura en invernadero, la alcanzó la combinación de gallinaza más HMA (Cuadro II). Los resultados indicaron la efectividad en campo de la gallinaza y $R$. intraradices de manera independiente o asociados, como fertilizantes biológicos, los cuales fueron semejantes comparados con la práctica de fertilización inorgánica tradicional recomendada. Por el contrario, en el arbusto lentisco (Pistacia lentiscus), Caravaca et al. (2002) encontraron una sinergia influenciada con la combinación entre $R$. intraradices y la adición de composta, donde obtuvieron la mayor altura de la planta después de un año.

La competitividad entre la fertilización biológica respecto a la inorgánica también ha sido indicada en otros estudios. En maíz forrajero, Fortis et al. (2009) reportaron los mayores rendimientos de forraje verde con vermicomposta (64t/ha) y biocomposta ( $56 \mathrm{t} / \mathrm{ha})$, superando al tratamiento de fertilización química con 200-100-100, el cual produjo 48 t/ha. Rodríguez et al. (2009) compararon los efectos de té de compost como fertilizante biológico vs. fertilización tradicional en tomate producido en invernadero, en dicho trabajo se registraron semejanzas en el diámetro, largo y peso del fruto y en el rendimiento entre los tratamientos. El rendimiento obtenido en el estudio superó casi el doble al promedio nacional (16.7 t/ha; SIAP 2014). La similitud de rendimiento de los cultivos entre la fertilización biológica e inorgánica, también ha sido señalada por otros autores. López et al. (2001) y Flores et al. (2007) compararon la adición de estiércol con la fertilización inorgánica en alfalfa y maíz, respectivamente y obtuvieron rendimientos semejantes entre ambos tipos de fertilización. Por otro lado, de igual forma, estudios comparativos entre inoculación con diferentes hongos micorrízicos y fertilización inorgánica, han demostrado respuesta similar en el rendimiento de sorgo, maíz y arroz (Díaz et al. 2008, Díaz et al. 2011, Orona et al. 2013).

Los contenidos foliares de $\mathrm{P}, \mathrm{Ca}, \mathrm{Mg}$ y de los elementos menores analizados, no fueron influenciados por los tratamientos de fertilización biológica o inorgánica en 2013 (Cuadro VII). Estos resultados

CUADRO VI. CARACTERÍSTICAS DE PLANTA, FRUTO Y RENDIMIENTO DE CALABACITA MEDIANTE GALLINAZA, MICORRIZA ARBUSCULAR Y FERTILIZACIÓN INORGÁNICA EN 2012 Y 2013

\begin{tabular}{lccccc}
\hline Tratamiento & \multirow{2}{*}{$\begin{array}{c}\text { Clorofila } \\
\text { (SPAD) }\end{array}$} & $\begin{array}{c}\text { Altura de } \\
\text { planta }(\mathrm{cm})\end{array}$ & \multicolumn{2}{c}{ Fruto } & \multirow{2}{*}{$\begin{array}{c}\text { Rendimiento } \\
\text { total }(\mathrm{t} / \mathrm{ha})\end{array}$} \\
\cline { 4 - 5 } & & & Diámetro $(\mathrm{mm})$ & Peso $(\mathrm{g})$ & \\
\hline Gallinaza $(3 \mathrm{t} / \mathrm{ha})$ & 36.0 & $62.4 \mathrm{ab}^{*}$ & 46.9 & 197 & 29.7 \\
HMA $(0.5 \mathrm{~kg} / \mathrm{ha})$ & 35.8 & $61.8 \mathrm{ab}$ & 47.8 & 199 & 30.0 \\
G + HMA & 35.2 & $59.3 \mathrm{~b}$ & 46.9 & 193 & 28.5 \\
120-90-00 & 36.2 & $65.8 \mathrm{a}$ & 46.5 & 195 & 30.8 \\
Significancia F & 0.075 & 0.040 & 0.24 & 0.64 & 0.07 \\
\hline
\end{tabular}

*Valores unidos con la misma letra en la misma columna no difieren (Tukey, $\mathrm{p} \leq 0.05$ ) $\mathrm{SPAD}=$ Valor del índice de clorofila con medidor Minolta SPAD 502, G= Gallinaza, HMA= Hongos micorrízicos arbusculares 
CUADRO VII. CONTENIDO NUTRICIONAL FOLIAR DE CALABACITA SOMETIDA A FERTILIZACIÓN BIOLÓGICA E INORGÁNICA EN 2013

\begin{tabular}{lccccccc}
\hline Tratamiento & $\mathrm{P}$ & $\mathrm{Cu}$ & $\mathrm{Zn}$ & $\mathrm{Mn}$ & $\mathrm{Fe}$ & $\mathrm{Ca}$ & $\mathrm{Mg}$ \\
\cline { 2 - 8 } & \multicolumn{7}{c}{$\mathrm{Mg} / \mathrm{kg}$} \\
\hline Gallinaza (3 t/ha) & 0.61 & 13.0 & 66.6 & 21.6 & 303 & $1.08 \mathrm{a}$ * & 0.83 \\
HMA (0.5 kg/ha) & 0.57 & 11.0 & 74.6 & 18.0 & 258 & $0.86 \mathrm{a}$ & 0.79 \\
G + HMA & 0.52 & 11.3 & 72.0 & 17.8 & 261 & $0.88 \mathrm{a}$ & 0.80 \\
120-90-00 & 0.51 & 11.0 & 73.3 & 21.3 & 301 & $1.09 \mathrm{a}$ & 0.84 \\
Significancia F & 0.682 & 0.522 & 0.814 & 0.314 & 0.114 & 0.048 & 0.192 \\
\hline
\end{tabular}

*Valores unidos con la misma letra no difieren (Tukey, $\mathrm{p} \leq 0.05$ )

$\mathrm{G}=$ Gallinaza, $\mathrm{HMA}=$ Hongos micorrízicos arbusculares

pueden asociarse con la respuesta similar del índice de clorofila entre los tratamientos obtenidos en 2012 y 2013 (Cuadro VI), variable que refleja de manera indirecta el estatus nutrimental del cultivo. Díaz et al. (2013) reportaron cantidades similares de N, P, Fe y Zn foliar en maíz inoculado con HMA y con fertilización inorgánica. Desgan y Bozkoylu (2007) determinaron que las concentraciones foliares de $\mathrm{N}, \mathrm{P}, \mathrm{K}, \mathrm{Ca}, \mathrm{Mg}, \mathrm{Fe}, \mathrm{Mn}, \mathrm{Zn}$ y $\mathrm{Cu}$ en calabacita, fueron semejantes o con pequeñas diferencias entre plantas con fertilización orgánica e inorgánica. Carvajal y Mera (2010) señalaron que a pesar de que los abonos orgánicos tienen menor cantidad de nutrimentos en comparación con los fertilizantes químicos, la disponibilidad de dichos elementos es más constante durante el desarrollo del cultivo por la mineralización gradual a que están sometidos. Sin embargo, es importante destacar que también el uso continuo de los abonos orgánicos puede ocasionar contaminación del suelo por metales pesados y aumento en los niveles de salinidad (Bouajila y Sanaa 2011). Además, los microorganismos también juegan un papel importante en la fertilidad ya que modifican las características químicas del suelo, las cuales están sujetas a las interacciones particulares entre sueloplanta-microorganismos, lo que da como resultado su capacidad amortiguadora de la fertilidad (Baera 1991, Mader et al. 2000).

Los inoculantes microbianos pueden ser capaces de sustituir o disminuir la fertilización inorgánica en los cultivos ya que promueven el crecimiento de las plantas, mejoran la disponibilidad y la absorción de nutrientes, así como la sanidad de las plantas (Adesemoye y Kloepper 2009). Otro aspecto importante a considerar con el uso de bioinoculantes en los cultivos, es el hecho de que se pueden reducir las emisiones de óxido nitroso $\left(\mathrm{N}_{2} \mathrm{O}\right)$ asociadas con la aplicación de fertilizantes nitrogenados (Calvo et al. 2013).

\section{CONCLUSIONES}

Los resultados de las características de la planta, el fruto y el rendimiento de la calabacita demostraron que el abonado con gallinaza procesada, la inoculación micorrízica y la combinación de ambos, arrojaron resultados similares a la fertilización inorgánica, lo que sugiere contar con prácticas agronómicas competitivas y más amigables con el ambiente.

\section{AGRADECIMIENTOS}

Se agradece el apoyo financiero de esta contribución a la Secretaría de Agricultura, Ganadería, Desarrollo Rural, Pesca y Alimentación mediante el proyecto "Impulso a nuevos productos y procesos de la bioeconomía y de investigación, transferencia de tecnología y uso de biocombustibles, biofertilizantes y abonos orgánicos". A Juan Olvera y Raúl Ortiz, por el apoyo en las labores de campo.

\section{REFERENCIAS}

Adesemoye A.O. y Kloepper J.W. (2009). Plant-microbes interactions in enhanced fertilizer-use efficiency. Appl. Microbiol. Biotechnol. 85, 1-12.

DOI: $10.1007 / \mathrm{s} 00253-009-2196-0$

Baera J. M. (1991). Vesicular-arbuscular mycorrhizae as modifiers of soil fertility. Adv. Soil Sci. 15, 1-40.

Bouajila K. y Sanaa M. (2011). Effects of organic amendments on soil physic-chemical and biological properties. J. Mater. Environ. Sci. 2, 485-490.

Calvo P., Watts D.B., Ames R.N., Kloepper J.W. y Torbert H.A. (2013). Microbial-based inoculants impact nitrous oxide emissions from an incubated soil medium containing urea fertilizers. J. Environ. Qual. 42, 704712. DOI: $10.2134 /$ jeq2012.0300 
Caravaca F., Barea J.M. y Roldán A. (2002). Synergistic influence of an arbuscular mycorrhizal fungus and organic amendment on Pistacia lentiscus L. seedlings afforested in a degraded semiarid soil. Soil Biol. Biochem. 34, 1139-1145.

DOI: $10.1016 / \mathrm{S} 0038-0717(02) 00047-0$

Carpio A.L., Davies F.T. y Arnold M.A. (2005). Arbuscular mycorrhizal fungi, organic and inorganic controlledrelease fertilizers: effect on growth and leachate of container-grown bush morning glory (Ipomoea carnea spp. fistulosa) under high production temperatures. J. Amer. Soc. Hort. Sci. 130, 131-139.

CERIB (2012). Paquetes tecnológicos para producción de cultivos. Centro de Investigación Regional Noreste. Campo Experimental Río Bravo, Instituto Nacional de Investigaciones Forestales, Agrícolas y Pecuarias, Tamaulipas, México, $40 \mathrm{pp}$.

Carvajal M.J. y Mera B.A. (2010). Fertilización biológica: técnica de vanguardia para el desarrollo agrícola sostenible. Producción 5, 78-96.

Davies F.T., Olalde P.V., Aguilera G.L., Alvarado M.J., Ferrera C.R. y Boutton T.W. (2002). Alleviation of drought stress of chile ancho pepper (Capsicum annuum L.) with arbuscular mycorrhiza indigenous to Mexico. Sci. Hort. 92, 347-359.

DOI: 10.1016/S0304-4238(01)00293-X

Desgan H.Y. y Bozkoylu A. (2007). Comparison of organic and synthetic-inorganic nutrition of soilless grown summer squash. Acta Hort. 747, 523-528.

DOI: 10.17660/ActaHortic.2007.747.68

Díaz F.A., Ortiz C.F.E., Lozano C.M., Aguado S.A. y Grageda C.O. (2013). Growth, mineral absorption and yield of maize inoculated with microbe strains. Afr. J. Agr. Res. 8, 3764-3769.

DOI: 10.5897/AJAR2012.6662

Díaz F.A., Pecina Q.V., Montes G.N., Jacques H.C. y Garza C.I. (2011). Impacto de inoculantes microbianos en sorgo cultivado bajo déficit de humedad en el suelo. En: Retos de la Investigación del Agua en México (S.O. Oswald, Ed.). Universidad Nacional Autónoma de México-Consejo Nacional de Ciencia y Tecnología, Ciudad de México, México. pp. 273-282.

Díaz F.A., Salinas G.J., Garza C.I. y Mayek P.N. (2008). Impacto de labranza e inoculación micorrízica arbuscular sobre la pudrición carbonosa y rendimiento de maíz en condiciones semiáridas. Rev. Fitotec. Mex. 31, 257-263.

Eghball B., Wienhold B.J., Guillery J.E. y Eigenberg R.A. (2002). Mineralization of manure nutrients. J. Soil Water Conservation 57, 470-473.

Flores M.J.P., Corral D.B. y Sapiens M.G. (2007). Mineralización de nitrógeno de biosólidos estabilizados con cal en suelos agrícolas. Terra Latinoamer. 25, 409-417.
Fortis H.M., Leos R.J., Preciado R.P. Orona C.I., García S.J., García H.J. y Orozco V.J. (2009). Aplicación de abonos orgánicos en la producción de maíz forrajero con riego por goteo. Terra Latinoamer. 27, 329-336.

Giovannetti M. y Mosse B. (1980). An evaluation of techniques for measuring vesicular-arbuscular mycorrhizal infection in roots. New Phytol. 84, 489-500.

DOI: 10.1111/J.1469-8137.1980.tb04556.x

Hue N.V. y Silva J.A. (2000). Organic soil amendments for sustainable agriculture. En: Plant nutrient management (J.A. Silva y R. Uchida, Eds.). Universidad de Hawaii, Honolulu, EUA, pp. 133-144.

Mader P., Endenhofer S., Boller T., Wienken A. y Niggli U. (2000). Arbuscular mycorrhizae in long-term field trail comparing low-input (organic, biological) and high-input (conventional) farming systems in a crop rotation. Biol. Fert. Soils 31, 150-156.

Masabni J. (2010). Cucurbits. En: Commercial vegetable crop guide. Texas Vegetable Growers' Handbook. Texas A and M University. Publ. B-6159. Brayan, Texas, EUA, pp 2.

López M.J., Díaz E.A., Martínez R.E. y Valdez C.R. (2001). Abonos orgánicos y su efecto en propiedades físicas y químicas del suelo y rendimiento de maíz. Terra Latinoamer. 19, 293-299.

Orona C.F., Lozano C.G., Tocuch C.M., Grageda C.O., Medina M.J., Díaz F.A., Ruiz S.E. y Soto R.J. (2013). Response of rice cultivation to biofertilizers in Campeche, Mexico. Agr. Sci. 4, 715-720.

DOI: $10.4236 /$ as.2013.412097

Phillips J.M. y Hayman D.S. (1970). Improved procedures for clearing roots and staining parasitic and vesicular arbuscular mycorrhizal fungi. Trans. Br. Mycol. Soc. $55,158-161$.

DOI: 10.1016/S0007-1536(70)80110-3

Plenecassangne A., Romero F.E. y López B.C. (1999). Manual de laboratorio para análisis de suelo, planta y agua. Instituto Nacional de Investigaciones Forestales, Agrícolas y Pecuarias. Gómez Palacio, Durango, México, $423 \mathrm{p}$.

Rabie G.H. (2005). Influence of arbuscular mycorrhizal fungi and kinetin on the response of mungbean plants to irrigation with seawater. Mycorrhiza 15, 225-230. DOI: 10.1007/s00572-004-0345-y

Ramírez M.M. (2012). Paquete tecnológico para la producción de chiles en Tamaulipas. Campo Experimental Las Huastecas, Instituto Nacional de Investigaciones Forestales, Agrícolas y Pecuarias, Tamaulipas, México, 11 pp. Rodríguez D.N., Cano R.P., Figueroa V.U., Favela C.E., Moreno R.A., Márquez H.C., Ochoa M.E. y Preciado R.P. (2009). Uso de abonos orgánicos en la producción de tomate en invernadero. Terra Lantinoamer. 27, 319-327. 
SIAP (2014). Sistema de Información Agroalimentaria y Pesquera. Secretaría de Agricultura, Ganadería, Desarrollo Rural, Pesca y Alimentación. [en línea] www.siap. gob.mx/cierre-de-la-produccion-agricola-por-cultivo/ 09/09/2014

Tahat M.M., Sijam K. y Othman R. (2010). Mycorrhizal fungi as a biocontrol agent. Plant Pathology J. 9, 198-207.

DOI: 10.3923/ppj.2010.198.207
Terry A.E., Leyva A. y Hernández A. (2005). Microorganismos benéficos como biofertilizantes eficientes para el cultivo del tomate (Lycopersicon esculentum). Rev. Colombiana Biotecnol. 7, 47-54.

Vásquez S.E. Lira S.R., Valdez A.L., Cárdenas F.A., Ibarra J.L. y González D. (2014). Respuesta de pepino a la fertilización biológica y mineral con y sin acolchado plástico en condiciones de casa sombra. Rev. Inter. Inv. Innov. Tecnol. 10, 1-11. 